\title{
Avaliação do armazenamento a frio sobre os compostos bioativos e as características físico-químicas e microbiológicas do suco de umbu pasteurizado
}

\author{
Evaluation of cold storage on the bioactive compounds and physical-chemical and \\ microbiological characteristics of pasteurized umbu juice
}

\section{Leilson de Oliveira Ribeiro', Sérgio Macedo Pontes², Ana Paula de Oliveira Ribeiro², Sidney Pacheco², Suely Pereira Freitas ${ }^{1}$, Virgínia Martins da Matta ${ }^{2 \star}$}

${ }^{1}$ Universidade Federal do Rio de Janeiro (UFRJ), Escola de Química, Rio de Janeiro/RJ - Brasil

2 Embrapa Agroindústria de Alimentos, Rio de Janeiro/RJ - Brasil

\section{${ }^{*}$ Corresponding Author}

Virgínia Martins da Matta, Embrapa Agroindústria de Alimentos, Avenida das Américas, 29501, CEP: 23020-470, Rio de Janeiro/RJ - Brasil, e-mail: virginia.matta@embrapa.br

Cite as: Evaluation of cold storage on the bioactive compounds and physical-chemical and microbiological characteristics of pasteurized umbu juice. Braz. J. Food Technol., v. 20, e2015095, 2017

\section{Resumo}

Este trabalho teve como objetivo avaliar o efeito do armazenamento a $6{ }^{\circ} \mathrm{C}$ nas características do suco de umbu pasteurizado. A pasteurização foi realizada a $86{ }^{\circ} \mathrm{C}$ por $25 \mathrm{~s}$ em um pasteurizador de superfície raspada, sendo o suco de umbu mantido a $6{ }^{\circ} \mathrm{C}$ por 90 dias. O suco pasteurizado atendeu ao padrão estabelecido pela legislação brasileira com contagem <3 NMP.g - $^{-1}$ para coliformes a $45^{\circ} \mathrm{C}$ e ausência de Salmonella sp. Nesta condição de processo, a pasteurização foi eficiente também para a redução de bactérias aeróbias mesófilas. O teor de compostos fenólicos totais e, em particular, da rutina, assim como a atividade antioxidante do suco, não foram afetados pelo tratamento térmico. Ao longo do armazenamento, apesar de não perceptível visualmente, os parâmetros de cor do suco variaram significativamente $(p<0,05)$. Apesar de pequena, também houve variação significativa $(p<0,05)$ nos teores dos compostos fenólicos e de carotenoides. Por outro lado, o suco de umbu se manteve microbiologicamente estável durante o armazenamento, sendo, portanto, possível sua conservação por um período de 90 dias sem o uso de aditivos, quando submetido à pasteurização e mantido sob refrigeração.

Palavras-chave: Spondias tuberosa; Vida de prateleira; Carotenoides; Compostos fenólicos.

\section{Abstract}

This work aimed to evaluate the effect of storage at $6^{\circ} \mathrm{C}$ on the characteristics of pasteurized umbu juice. Pasteurization was carried out at $86{ }^{\circ} \mathrm{C}$ for $25 \mathrm{~s}$ in a scraped surface heat exchanger and storage at $6{ }^{\circ} \mathrm{C}$ for 90 days. The pasteurized umbu juice conformed to the standards established by Brazilian legislation, presenting $<3 \mathrm{MPN} . \mathrm{g}^{-1}$ for $\mathrm{coliforms}$ at $45^{\circ} \mathrm{C}$ and absence of Salmonella sp. Under these processing conditions, pasteurization was also efficient in reducing the mesophilic aerobic bacteria. The phenolic compounds content, and specifically that of rutin, and the antioxidant activity of the juice were not affected $(p<0.05)$ by the heat treatment. Although not visually perceptible, the colour parameters of the umbu juice were significantly affected by storage $(p<0.05)$. Although small, there was also significant variation $(p<0.05)$ in the phenolic compounds and carotenoid contents. On the other hand, the umbu juice remained microbiologically stable during storage, showing that it is possible to preserve it for a period of 90 days without the use of preservatives when submitted to pasteurization and kept under refrigeration.

Keywords: Spondias tuberosa; Shelf life; Carotenoids; Phenolic compounds. 
Avaliação do armazenamento a frio sobre os compostos bioativos e as características físico-químicas e microbiológicas do suco de umbu pasteurizado

Ribeiro, L. O. et al.

\section{Introdução}

O Brasil é o terceiro maior produtor mundial de frutas, superado apenas pela China e Índia (REETZ et al., 2015). Dentre as suas regiões produtoras de frutas, pode-se destacar a região Nordeste, com frutos ainda pouco explorados tanto do ponto de vista comercial quanto científico, visto que muitos destes possuem compostos importantes para a saúde humana.

O umbu (Spondias tuberosa Arruda Câmara) é um exemplo de fruto pouco explorado. Este se destaca no cenário brasileiro por se apresentar como fonte de renda para as famílias do semiárido e por conter compostos como carotenoides e fenólicos, todos com potencial atividade biológica. De acordo com dados do IBGE (2014), em 2013 foram produzidas cerca de sete mil toneladas do fruto. Apesar deste potencial, sua comercialização ainda é bem artesanal, o que favorece as perdas e compromete sua qualidade microbiológica.

Por se tratar de um fruto nativo e pouco conhecido nas demais regiões do país, chegando a ser considerado exótico, em vários trabalhos têm sido propostos novos produtos à base do fruto visando à sua agregação de valor e inserção no mercado. Produtos como néctar misto (MATTIETTO et al., 2007), fermentado (PAULA et al., 2012) e outros já foram estudados, apesar de o principal produto comercializado ainda ser a polpa congelada.

A elaboração de produtos na forma de polpa congelada, por se apresentar como um produto de fácil obtenção, é uma das alternativas mais utilizadas por pequenos produtores. No entanto, quando se pensa em produtos que atendam a requisitos do mercado consumidor, como a praticidade, este tipo de produto não corresponde às expectativas. Assim, um suco integral armazenado sob refrigeração, que não necessita ser descongelado, pode atender a esse mercado, além de ser uma forma de agregação de valor ao fruto.

Este tipo de produto necessita do uso de processos para a sua estabilização, principalmente microbiológica, para que possa ser armazenado por um longo período. Uma forma bastante utilizada pela indústria de alimentos para prolongar a vida útil de seus produtos é o emprego do calor. A pasteurização é um dos processos mais aplicados na conservação de polpas e sucos de frutas, pois a combinação apropriada da temperatura e do tempo permite destruir microrganismos e inativar enzimas e, com isso, aumentar a vida útil do produto (FELLOWS, 2006). No entanto, em condições impróprias, o binômio pode comprometer a cor e demais características sensoriais e nutricionais.

Oliveira et al. (2011) avaliaram o efeito da pasteurização da polpa de umbu sobre a sua qualidade microbiológica, suas características físico-químicas e cor instrumental. Porém, com relação aos compostos bioativos, pouco foi estudado, sendo apenas o teor de carotenoides totais objeto de acompanhamento.

Desta forma, o objetivo deste trabalho foi avaliar o efeito do armazenamento a frio do suco de umbu pasteurizado sobre a sua qualidade microbiológica, cor instrumental, características físico-químicas e compostos bioativos, por um período de 90 dias.

\section{Material e métodos}

A matéria-prima utilizada neste trabalho foi a polpa de umbu comercial congelada, adquirida diretamente da indústria produtora no Estado da Bahia, sendo transportada e mantida congelada até o momento de realização dos experimentos que, neste trabalho, foi denominada de suco.

O suco de umbu foi pasteurizado a $86{ }^{\circ} \mathrm{C}$ por 25 s (RIBEIRO, 2014) e envasado em uma câmara de envase ultralimpo em garrafas de vidro transparente de $200 \mathrm{~mL}$, previamente sanitizadas com hipoclorito de sódio (20 mL. $\mathrm{kg}^{-1}$ ) e fechadas com tampas do tipo garra-torção. O suco pasteurizado foi armazenado sob refrigeração a $6{ }^{\circ} \mathrm{C}$ em incubadora do tipo BOD (Biochemical Oxigen Demand) durante 90 dias. As amostras foram retiradas a cada 15 dias para avaliação da qualidade microbiológica e da cor instrumental e a cada 30 dias para avaliação dos parâmetros físico-químicos, compostos bioativos e atividade antioxidante, conforme descrito a seguir.

As amostras foram analisadas para detecção de Salmonella, coliformes a $45^{\circ} \mathrm{C}$, contagem padrão em placas de aeróbias mesófilas e psicrotróficas, contagem de fungos filamentosos e leveduras, segundo as metodologias recomendadas pela American Public Health Association (APHA, 2001).

A análise instrumental de cor foi realizada em colorímetro (ColorQuest XE, Hunterlab) utilizando o sistema CIELab/CIELCH de coordenadas $L^{*}$, $a^{*}, b^{*}$, e ângulo Hue (h) com abertura de $0,375 \mathrm{~mm}$ de diâmetro, com iluminante D65/10 (FERREIRA, 1981), onde $L^{*}=$ luminosidade $(0=$ preto e $100=$ branco $) ; a^{*}(-80$ até zero $=$ verde, do zero ao $+100=$ vermelho $) ; b^{*}(-100$ até zero $=$ azul, do zero ao $+70=$ amarelo); $h^{\circ}$ ângulo Hue $=\arctan \left(b^{*} / a^{*}\right)$.

Os sólidos solúveis foram determinados por meio de leitura direta em refratômetro digital (ATAGO) segundo o método 932.14 da Horwitz (2000). O pH e acidez titulável foram determinados em titulador automático (Metrohm, 785 DMP Tritino), de acordo com as metodologias descritas pela AOAC (HORWITZ, 2000), respectivamente 973.41 e 942.15 .

Os carotenoides foram extraídos utilizando a metodologia proposta por Rodriguez-Amaya (1999), com auxílio de acetona P.A., por meio de extração exaustiva, ou seja, até que não se observasse mais coloração amarela na amostra pesada, e quantificados por método espectrofotométrico a $453 \mathrm{~nm}$. O perfil de carotenoides 
Ribeiro, L. O. et al.

foi analisado por cromatografia líquida de alta eficiência (CLAE) com separação em fase reversa conforme metodologia proposta por Pacheco et al. (2014), sendo a corrida cromatográfica realizada em cromatógrafo líquido Alliance 2695 da Waters $^{\circledR}$, com detector de arranjo de diodos (DAD) 2996 da Waters $^{\circledR}$ a $450 \mathrm{~nm}$, coluna YMC Carotenoid S-3 ${ }^{\circledR}(4,6 \times 250 \mathrm{~mm})$; fase móvel $(A)$ metanol e (B) éter metil-terc-butílico em eluição gradiente; fluxo $0,8 \mathrm{~mL} \mathrm{~min}^{-1}$, temperatura da coluna $33^{\circ} \mathrm{C}$ e volume de injeção $15 \mu \mathrm{L}$. A identificação dos carotenoides foi realizada por comparação dos tempos de retenção e espectros de absorção UV/Vis com padrões. A quantificação foi realizada por padronização externa. Para melhor resolução do cromatograma, os extratos contendo os carotenoides foram saponificados com auxílio de solução metanólica de hidróxido de potássio 10\%, de acordo com Pacheco et al. (2014).

Os compostos fenólicos totais foram determinados de acordo com método descrito por Singleton e Rossi (1965) modificado por Georgé et al. (2005), sendo extraídos em acetona $70 \%$ por 30 minutos sob agitação. O ácido gálico foi utilizado como padrão analítico, sendo os resultados expressos em ácido gálico equivalente por $100 \mathrm{~g}$ de amostra (mg.100 g ${ }^{-1}$ ).

A determinação dos flavonoides foi realizada por CLAE, com separação em fase reversa e detecção por DAD, a partir do método de extração de isoflavonas da AOAC (HORWITZ, 2005) (2001.10), adaptado por Godoy et al. (2013). A extração em metanol $80 \%$ ocorreu por duas horas a $65^{\circ} \mathrm{C}$. Depois do resfriamento da amostra, foram adicionados $3 \mathrm{~mL}$ de $\mathrm{NaOH}$ 2M e ela foi submetida a agitação por 10 minutos com posterior adição de ácido acético. A amostra foi centrifugada a 6000 rpm por 10 minutos e foi feita a filtração direta do sobrenadante em vials. A corrida cromatográfica foi realizada em cromatógrafo líquido Alliance 2695 da Waters ${ }^{\circledR}$, com DAD 2996 da Waters $^{\circledR}$ a $260 \mathrm{~nm}$, coluna Thermo BDS Hypersil C18 (100X 4,6 mm 2,4 um); fase móvel (A) ácido fórmico 1\% e (B) acetonitrila em eluição gradiente; fluxo 1,3 $\mathrm{mL}$ min $^{-1}$; temperatura da coluna $45^{\circ} \mathrm{C}$; volume de injeção $25 \mu \mathrm{L}$. A quantificação foi realizada por padronização externa, por meio da elaboração de uma curva padrão contendo padrões de epicatequina, daidzina, glicetina, genistina, naringina, rutina, miricetina, hesperidina, neo-hesperidina, daidzeína, diosmina, gliciteína, quercetina, genisteína, naringinina, hesperetina, kaempferol e isovitexina, flavonoides comumente encontrados em vegetais (IGNAT et al., 2011; NACZK; SHAHIDI, 2006, 2004).

A atividade antioxidante foi determinada pelo método de redução do radical $\mathrm{ABTS}+\bullet$, de acordo com $\mathrm{Re}$ et al. (1999) e os resultados foram expressos em $\mu$ mol Trolox por grama de amostra. Para tal, as amostras foram extraídas, em primeiro lugar, com metanol $50 \%$ por 1 hora e, em seguida, centrifugadas para a separação do sobrenadante.
O precipitado foi novamente extraído com acetona 70\% nas mesmas condições citadas anteriormente. Os extratos foram combinados e adicionados de água destilada para completar o volume de $25 \mathrm{~mL}$.

Os resultados expressos como média \pm desvio padrão das determinações realizadas em triplicata foram tratados estatisticamente por análise de variância (ANOVA), considerando o teste de Tukey para avaliar diferenças significativas entre as médias, utilizando-se o intervalo de confiança de 95\%, por meio do software Statistica 7.0 (Statsoft Inc., Tulsa, OK, EUA).

\section{Resultados e discussão}

\subsection{Pasteurização}

Na Tabela 1 são mostrados os parâmetros avaliados no suco de umbu antes (suco controle) e após a pasteurização, onde se verifica que o suco de umbu pasteurizado atendeu à legislação brasileira (BRASIL, 2001) quanto à qualidade microbiológica, a qual estabelece ausência de Salmonela sp e contagem $<3 \mathrm{NMP} . \mathrm{g}^{-1}$ para coliformes a $45^{\circ} \mathrm{C}$. O efeito positivo do calor sobre a carga microbiana de sucos de frutas já tem sido reportado por vários estudos, como, por exemplo, os resultados reportados por Mena et al. (2013). Estes autores observaram redução significativa de bactérias aeróbias mesófilas em suco de romã tratado a temperaturas de $65^{\circ} \mathrm{C}$ a $95^{\circ} \mathrm{C}$ por 30 a 60 segundos. Outro exemplo é o estudo realizado por Xu et al. (2015), que avaliaram o efeito da pasteurização de suco de laranja com pimenta a $110^{\circ} \mathrm{C}$ por 8,6 segundos. Depois do tratamento térmico, observou-se redução de 4 ciclos logarítmicos de fungos filamentosos e leveduras e de bactérias aeróbias mesófilas.

Analisando-se os resultados de cor, foram observadas alterações significativas $(p<0,05)$ no suco de umbu, confirmando o efeito negativo, característico do tratamento térmico, sobre a cor de sucos de fruta. Este comportamento também foi reportado por Santhirasegaram et al. (2015), ao avaliarem o efeito da pasteurização convencional sobre a cor do suco de manga cultivar Chokanan, como também por Keenan et al. (2012), que trataram termicamente um smoothie de frutas.

Os carotenoides, como se sabe, têm influência direta na cor dos alimentos, o que se verifica também no suco de umbu. Com exceção da zeaxantina e do 13-cis- $\beta$-caroteno, todos os outros carotenoides presentes no suco sofreram degradação com a pasteurização. Os carotenoides que apresentaram maior degradação após a pasteurização foram luteína (37,5\%), $\beta$-criptoxantina (10,8\%) e $\beta$-caroteno $(6,7 \%)$.

Gama e Sylos (2007), ao estudarem a pasteurização de suco de laranja em temperaturas entre $95^{\circ} \mathrm{C}$ e $105^{\circ} \mathrm{C}$ por 10 segundos, observaram a perda de violaxantina (38\%),

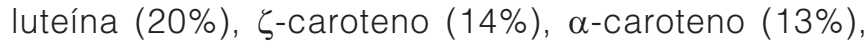


Avaliação do armazenamento a frio sobre os compostos bioativos e as características físico-químicas e microbiológicas do suco de umbu pasteurizado

Ribeiro, L. O. et al.

Tabela 1. Características físico-químicas, compostos bioativos, atividade antioxidante, cor instrumental e qualidade microbiológica do suco de umbu antes e após a pasteurização.

\section{Parâmetro}

Salmonella sp (ausência em $25 \mathrm{~g}$ )

Coliformes a $45^{\circ} \mathrm{C}\left(\mathrm{NMP} . \mathrm{g}^{-1}\right)$

Fungos e leveduras (UFC. $\mathrm{g}^{-1}$ )

Contagem aeróbios mesófilos (UFC. $\mathrm{g}^{-1}$ )

$\mathrm{pH}$

Sólidos solúveis ( ${ }^{\circ}$ Brix)

Acidez titulável $\left(\mathrm{g} .100 \mathrm{~g}^{-1}\right)$

Fenólicos totais (mg ac. Gal.100 g $\mathrm{g}^{-1}$ )

Rutina (mg.100 g-1)

Quercetina (mg.100 $\mathrm{g}^{-1}$ )

Atividade antioxidante ( $\mu \mathrm{mol}$ Trolox. $\left.\mathrm{g}^{-1}\right)$

Carotenoides totais $\left(\mu \mathrm{g} .100 \mathrm{~g}^{-1}\right)$

Luteína $\left(\mu \mathrm{g} .100 \mathrm{~g}^{-1}\right)$

Zeaxantina $\left(\mu \mathrm{g} .100 \mathrm{~g}^{-1}\right)$

$\beta$-criptoxantina $\left(\mu \mathrm{g} .100 \mathrm{~g}^{-1}\right)$

$\beta$-caroteno $\left(\mu \mathrm{g} 100 \mathrm{~g}^{-1}\right)$

13-cis- $\beta$-caroteno $\left(\mu \mathrm{g} .100 \mathrm{~g}^{-1}\right)$

9-cis- $\beta$-caroteno $\left(\mu \mathrm{g} .100 \mathrm{~g}^{-1}\right)$

L*

$a^{*}$

$b^{*}$

$\mathrm{H}^{\circ}$

\begin{tabular}{|c|c|}
\hline Controle* & Suco pasteurizado \\
\hline Ausência & Ausência \\
\hline$<3$ & $<3$ \\
\hline$<1,0 \times 10^{1}$ & $<1,0 \times 10^{1}$ \\
\hline $3,5 \times 10^{2}$ & $<1,0 \times 10^{1}$ \\
\hline $2,49 \pm 0,01^{a}$ & $2,51 \pm 0,01^{b}$ \\
\hline $5,0 \pm 0,1^{a}$ & $5,0 \pm 0,1^{a}$ \\
\hline $1,70 \pm 0,00^{a}$ & $1,67 \pm 0,01^{b}$ \\
\hline $93,13 \pm 3,77^{a}$ & $191,34 \pm 4,51^{a}$ \\
\hline $88,22 \pm 2,10^{a}$ & $82,40 \pm 2,05^{a}$ \\
\hline $2,97 \pm 0,01^{a}$ & $2,32 \pm 0,06^{b}$ \\
\hline $11,04 \pm 0,33^{a}$ & $10,74 \pm 0,13^{a}$ \\
\hline $27,35 \pm 2,91^{a}$ & $309,58 \pm 8,59^{b}$ \\
\hline $13,33 \pm 0,58^{a}$ & $8,33 \pm 0,58^{b}$ \\
\hline $4,33 \pm 0,58^{a}$ & $4,33 \pm 0,58^{a}$ \\
\hline $58,33 \pm 0,58^{a}$ & $52,00 \pm 2,65^{b}$ \\
\hline $70,33 \pm 1,53^{a}$ & $159,00 \pm 3,00^{b}$ \\
\hline $5,33 \pm 0,58^{a}$ & $5,00 \pm 0,00^{a}$ \\
\hline $23,33 \pm 0,58^{a}$ & $22,00 \pm 0,00^{b}$ \\
\hline $47,98 \pm 0,13^{a}$ & $46,37 \pm 0,13^{b}$ \\
\hline$-0,31 \pm 0,04^{a}$ & $0,26 \pm 0,04^{b}$ \\
\hline $12,76 \pm 0,07^{a}$ & $11,84 \pm 0,07^{b}$ \\
\hline $91,41 \pm 0,19^{a}$ & $88,76 \pm 0,21^{b}$ \\
\hline
\end{tabular}

${ }^{*}$ Controle - suco de umbu antes da pasteurização. Letras iquais na mesma linha não apresentam diferença significativa entre os resultados $(p>0,05)$. Resultados expressos em média \pm desvio padrão. $L^{*}$ - luminosidade instrumental; $a^{*}$ - intensidade de cor vermelha; $b^{*}$ - intensidade de cor amarela; $\mathrm{H}^{\circ}$ - ângulo Hue.

$\beta$-caroteno (11\%) e zeaxantina ( $9 \%)$. Estes autores reportam que, como os carotenoides são considerados ativos contra a degeneração macular e cataratas relacionadas com a idade, se faz importante o estudo das condições operacionais da pasteurização para que se tenha não somente a garantia da qualidade microbiológica, como também a preservação destes compostos biologicamente ativos presentes no suco em questão.

Em estudo realizado por Oliveira et al. (2012), no qual foi avaliada a pasteurização de pêssego a $90^{\circ} \mathrm{C}$ por 5 minutos, também foram observadas alterações no perfil de carotenoides da fruta. Os autores relataram decréscimo nos níveis de zeaxantina e degradação total da $\beta$-criptoxantina, porém os níveis de luteína e $\beta$-caroteno permaneceram inalterados após a pasteurização. De acordo com os autores, a degradação dos pigmentos pode ocorrer devido a reações de oxidação que são fortemente influenciadas pela temperatura de pasteurização.

Por outro lado, os compostos fenólicos totais e a atividade antioxidante do suco de umbu não foram alterados significativamente após a pasteurização, comportamento que também foi observado por Oliveira et al. (2012) na pasteurização do pêssego a $90{ }^{\circ} \mathrm{C}$ por 5 minutos.

Entre os compostos fenólicos identificados neste trabalho não se observou diferença significativa para a rutina, flavonoide presente em maior concentração, enquanto que a pasteurização afetou significativamente $(p<0,05)$ o teor de quercetina. Este comportamento pode estar associado à estrutura química desses flavonoides, uma vez que a rutina é um derivado glicosilado da quercetina, o que pode lhe conferir maior estabilidade térmica.

\subsection{Estudo da estabilidade}

Na Tabela 2 estão apresentados os resultados da avaliação microbiológica do suco de umbu pasteurizado durante o período de armazenamento sob refrigeração $\left(6^{\circ} \mathrm{C}\right)$, em que se verificou a ausência de Salmonella sp e de coliformes a $45^{\circ} \mathrm{C}$ nos tempos 0 e 90 dias de armazenamento. Como as amostras, tanto do suco pasteurizado quanto do suco controle, estavam isentas desses microrganismos (o que confirma a adoção de Boas Práticas de Fabricação), não foi necessário analisar estes microrganismos em todos os tempos do armazenamento, sendo, por isso, repetido apenas no tempo final como uma forma de validar o resultado inicial. Estes resultados estão de acordo com o padrão estabelecido pela legislação brasileira vigente (BRASIL, 2001).

Os resultados mostram que o suco de umbu permaneceu estável ao longo de 60 dias, porém observou-se, no período de 75 dias, um pequeno crescimento de fungos 
Avaliação do armazenamento a frio sobre os compostos bioativos e as características físico-químicas e microbiológicas do suco de umbu pasteurizado

Ribeiro, L. O. et al.

filamentosos e leveduras e de bactérias psicrotróficas. Como não foi verificado crescimento no período posterior, correspondente aos 90 dias, isto sugere que pode ter ocorrido uma contaminação na garrafa daquela amostra específica, pois o suco foi acondicionado em garrafas individuais. Além disso, mesmo que estes valores tivessem sido verificados na amostra analisada no tempo final do armazenamento, o produto ainda estaria apto para o consumo, pois os valores detectados para ambas as classes de microrganismos são muito baixos e não configuram que o produto esteja deteriorado. De acordo com o Padrão de Identidade e Qualidade de Polpas de frutas (PIQ) do Ministério da Agricultura, Pecuária e Abastecimento, produtos pasteurizados, como os sucos integrais, que apresentam as mesmas características de polpas de frutas, podem apresentar contagem de fungos filamentosos e leveduras de até $10^{3}$ UFC.g ${ }^{-1}$ (BRASIL, 2000). Portanto, os resultados obtidos neste trabalho não configuram a instabilidade microbiológica do suco de umbu ao longo do seu armazenamento.
Com relação às características físico-químicas do suco de umbu (Tabela 3) mantido sob refrigeração $\left(6^{\circ} \mathrm{C}\right)$, observou-se pouca alteração durante o seu armazenamento.

$\mathrm{O}$ pH do suco aos 90 dias de armazenamento apresentou um ligeiro aumento $(p<0,05)$, enquanto que o teor de sólidos solúveis não sofreu alteração significativa $(p<0,05)$ durante o mesmo período. A acidez do suco de umbu, por sua vez, apresentou um pequeno aumento que foi diferente ao nível de 5\% de significância. No entanto, as alterações observadas são pequenas, não apresentando influência sobre a qualidade do produto e, além disso, em escala industrial podem ser consideradas irrelevantes. Tal comportamento também foi evidenciado por Guan et al. (2016) ao avaliarem a estabilidade físico-química do suco de manga tratado termicamente a $90^{\circ} \mathrm{C}$ por 60 segundos e armazenado sob refrigeração por um período de 60 dias.

Na Tabela 4 está mostrado o comportamento dos parâmetros de cor $\left(\mathrm{L}^{*}, \mathrm{~b}^{*} \mathrm{e} \mathrm{H}^{\circ}\right)$ durante o armazenamento do suco de umbu pasteurizado.

Tabela 2. Avaliação microbiológica do suco de umbu pasteurizado armazenado a $6{ }^{\circ} \mathrm{C}$ por 90 dias

\begin{tabular}{lccccccc}
\multicolumn{1}{c}{ Parâmetro } & \multicolumn{7}{c}{ Tempo de armazenamento (dias) } \\
\cline { 2 - 8 } & $\mathbf{0}$ & $\mathbf{1 5}$ & $\mathbf{3 0}$ & $\mathbf{4 5}$ & $\mathbf{6 0}$ & $\mathbf{7 5}$ & $\mathbf{9 0}$ \\
Coliformes a $45^{\circ} \mathrm{C}\left(\mathrm{NMP}_{\mathrm{y}}{ }^{-1}\right)$ & $<3$ & $\mathrm{nr}$ & $\mathrm{nr}$ & $\mathrm{nr}$ & $\mathrm{nr}$ & $\mathrm{nr}$ & $<3$ \\
Salmonella sp $(25 \mathrm{~g}$ ) & $\mathrm{Aus}$ & $\mathrm{nr}$ & $\mathrm{nr}$ & $\mathrm{nr}$ & $\mathrm{nr}$ & $\mathrm{nr}$ & $\mathrm{Aus}$ \\
Fungos filamentosos e leveduras (UFC. $\mathrm{g}^{-1}$ ) & $<10^{1}$ & $<10^{1}$ & $<10^{1}$ & $<10^{1}$ & $<10^{1}$ & $4 \times 10^{2}$ & $<10^{1}$ \\
Bactérias aeróbias psicrotróficas (UFC. $\mathrm{g}^{-1}$ ) & $<10^{1}$ & $<10^{1}$ & $<10^{1}$ & $<10^{1}$ & $<10^{1}$ & $5 \times 10^{1}$ & $<10^{1}$ \\
\hline
\end{tabular}

nr - não realizado; Aus - ausência.

Tabela 3. Características físico-químicas do suco de umbu pasteurizado armazenado a $6{ }^{\circ} \mathrm{C}$ por 90 dias.

\begin{tabular}{|c|c|c|c|c|}
\hline Amostra & Tempo (dias) & pH & $\begin{array}{c}\text { Sólidos solúveis } \\
\text { ( }{ }^{\circ} \text { Brix) }\end{array}$ & $\begin{array}{c}\text { Acidez } \\
\text { (g ác. cítrico.100 g-1) }\end{array}$ \\
\hline Controle* $^{*}$ & - & $2,49 \pm 0,01^{a}$ & $5,0 \pm 0,1^{a}$ & $1,70 \pm 0,00^{a}$ \\
\hline T0 & 0 & $2,51 \pm 0,01^{a}$ & $5,0 \pm 0,1^{a}$ & $1,67 \pm 0,01^{b}$ \\
\hline $\mathrm{T} 2$ & 30 & $2,51 \pm 0,02^{a}$ & $5,0 \pm 0,0^{a}$ & $1,67 \pm 0,01^{b}$ \\
\hline T4 & 60 & $2,52 \pm 0,01^{a}$ & $5,1 \pm 0,1^{a}$ & $1,74 \pm 0,00^{c}$ \\
\hline T6 & 90 & $2,57 \pm 0,01^{b}$ & $5,0 \pm 0,0^{a}$ & $1,72 \pm 0,01^{d}$ \\
\hline
\end{tabular}

${ }^{*}$ Controle - suco de umbu antes da pasteurização. Letras iguais na mesma coluna não apresentam diferença significativa entre os resultados $(p<0,05)$. Resultados expressos em média \pm desvio padrão.

Tabela 4. Parâmetros de cor do suco de umbu pasteurizado armazenado a $6{ }^{\circ} \mathrm{C}$ por 90 dias.

\begin{tabular}{ccccc} 
Amostra & Tempo (dias) & $\mathbf{L}^{*}$ & $\mathbf{b}^{*}$ & $\mathbf{H}^{\mathbf{*}}$ \\
Controle $^{*}$ & - & $47,98 \pm 0,13^{\mathrm{a}}$ & $12,76 \pm 0,07^{\mathrm{a}}$ & $91,41 \pm 0,19^{\mathrm{a}}$ \\
T0 & 0 & $46,37 \pm 0,13^{\mathrm{b}}$ & $11,84 \pm 0,07^{\mathrm{b}}$ & $88,76 \pm 0,21^{\mathrm{b}, \mathrm{d}}$ \\
T1 & 15 & $46,63 \pm 0,13^{\mathrm{b}, \mathrm{d}}$ & $11,80 \pm 0,16^{\mathrm{b}}$ & $89,64 \pm 0,44^{\mathrm{c}}$ \\
T2 & 30 & $46,47 \pm 0,08^{\mathrm{b}}$ & $11,56 \pm 0,08^{\mathrm{c}}$ & $89,64 \pm 0,44^{\mathrm{c}}$ \\
T3 & 45 & $45,63 \pm 0,18^{\mathrm{c}}$ & $11,38 \pm 0,11^{\mathrm{c}}$ & $88,53 \pm 0,20^{\mathrm{d}}$ \\
T4 & 60 & $46,35 \pm 0,04^{\mathrm{b}}$ & $11,34 \pm 0,07^{\mathrm{c}}$ & $87,98 \pm 0,08^{\mathrm{d}}$ \\
T5 & 75 & $45,92 \pm 0,14^{\mathrm{c}}$ & $10,90 \pm 0,11^{\mathrm{d}}$ & $87,11 \pm 0,18^{\mathrm{e}}$ \\
T6 & 90 & $46,85 \pm 0,15^{\mathrm{d}}$ & $13,45 \pm 0,07^{\mathrm{e}}$ & $89,32 \pm 0,18^{\mathrm{b}, \mathrm{c}}$ \\
\hline
\end{tabular}

${ }^{*}$ Controle - suco de umbu antes da pasteurização. Letras iguais na mesma coluna não apresentam diferença significativa entre os resultados $(p>0,05)$. Resultados expressos como média \pm desvio padrão. $L^{*}$ - luminosidade instrumental; $b^{*}$ - intensidade de cor amarela; $H^{\circ}-$ ângulo Hue. 
Avaliação do armazenamento a frio sobre os compostos bioativos e as características físico-químicas e microbiológicas do suco de umbu pasteurizado

Ribeiro, L. O. et al.

Tabela 5. Carotenoides, compostos fenólicos e atividade antioxidante do suco de umbu pasteurizado armazenado a $6^{\circ} \mathrm{C}$ por 90 dias

\begin{tabular}{|c|c|c|c|c|}
\hline Amostra & Tempo (dias) & $\begin{array}{c}\text { CT } \\
\left(\mu \mathrm{g} \beta \text {-caroteno.100 } \mathrm{g}^{-1}\right)\end{array}$ & $\begin{array}{c}\mathrm{FT} \\
\left.\text { (mg ac. gálico.100 } \mathrm{g}^{-1}\right)\end{array}$ & $\begin{array}{c}\text { AA } \\
\left(\mu \mathrm{mol} \text { Trolox. } \mathrm{g}^{-1}\right)\end{array}$ \\
\hline Controle* & - & $327,35 \pm 2,91^{a}$ & $193,10 \pm 3,77^{a}$ & $11,04 \pm 0,33^{a}$ \\
\hline TO & 0 & $309,58 \pm 8,59^{b}$ & $191,34 \pm 4,51^{a}$ & $10,74 \pm 0,13^{a}$ \\
\hline $\mathrm{T} 2$ & 30 & $282,20 \pm 8,04^{\circ}$ & $177,75 \pm 2,17^{b}$ & $9,48 \pm 0,37^{b}$ \\
\hline T4 & 60 & $285,37 \pm 1,51^{c}$ & $161,65 \pm 2,44^{c}$ & $9,13 \pm 0,58^{b}$ \\
\hline T6 & 90 & $291,68 \pm 3,14^{c}$ & $170,80 \pm 2,84^{b}$ & $9,19 \pm 0,24^{b}$ \\
\hline
\end{tabular}

${ }^{*}$ Controle - suco de umbu antes da pasteurizacão. CT - carotenoides totais; FT - fenólicos totais; AA - atividade antioxidante. Letras iguais na mesma coluna não apresentam diferença significativa entre os resultados ( $p>0,05)$. Resultados expressos como média \pm desvio padrão.

Verificou-se alteração significativa nos parâmetros de cor ( $p<0,05)$, não visível a olho nu, sendo a maior delas observada aos 90 dias do armazenamento. Neste tempo, houve um aumento nos parâmetros de cor, comportamento contrário ao que vinha ocorrendo, ou seja, escurecimento do suco ao longo do armazenamento, possivelmente pela degradação dos pigmentos do suco de umbu.

Embora tenha sido observado aumento nos parâmetros de cor no tempo de armazenamento de 90 dias, verificou-se que a luminosidade e o ângulo Hue foram sempre menores quando comparados à amostra controle, mostrando que a pasteurização e o armazenamento atuaram, de forma negativa, sobre a cor do suco de umbu. Este comportamento também foi evidenciado por Laorko et al. (2013), que observaram o escurecimento do suco de abacaxi clarificado por microfiltração armazenado por 6 meses a $4^{\circ} \mathrm{C}, 27^{\circ} \mathrm{C}$ e $37^{\circ} \mathrm{C}$. Para eles, o escurecimento do produto resultou de reações não enzimáticas ocorridas ao longo do armazenamento, tais como oxidação e polimerização de compostos bioativos. Eles verificaram que o escurecimento foi menos acentuado a $4{ }^{\circ} \mathrm{C}$. Guan et al. (2016), ao avaliarem o armazenamento do suco de manga pasteurizado por 60 dias à temperatura ambiente e sob refrigeração, também verificaram, como esperado, uma maior estabilidade dos parâmetros de cor à temperatura mais baixa, comprovando a contribuição da temperatura para a estabilidade do produto.

Para o teor de carotenoides do suco de umbu (Tabela 5), também se observou degradação destes compostos nos primeiros 30 dias de armazenamento. No entanto, após este período, até o período final da avaliação (90 dias de armazenamento), não foram observadas perdas significativas $(p>0,05)$ destes pigmentos.

Este comportamento pode ser explicado pela cinética de degradação dos carotenoides. Segundo Tsimidou e Biliaderis (1997), a degradação dos carotenoides presentes em produtos aquosos, como o suco de umbu, segue o comportamento de uma reação de primeira ordem. Isto também foi observado por Ferreira e Rodriguez-Amaya (2008) ao estudarem a degradação do licopeno e do $\beta$-caroteno em sistemas modelo de baixa umidade e aquoso, na presença e ausência de luz. Eles mostraram que a degradação dos carotenoides seguiu uma cinética de primeira ordem em todas as condições estudadas.

Assim, para o suco de umbu armazenado sob refrigeração, é também provável que se trate de um modelo cinético de primeira ordem, em que a velocidade da reação foi diretamente influenciada pelo teor dos carotenoides, que diminuiu significativamente aos 30 dias, tornando a velocidade da reação de degradação lenta o suficiente, para que não se observasse diferença significativa após este período, corroborando com a estabilidade do pigmento.

Outros parâmetros importantes foram observados durante o estudo da estabilidade do suco de umbu como o conteúdo de compostos fenólicos totais e a atividade antioxidante (Tabela 5). Os resultados mostram que houve diferença significativa $(p<0,05)$ em relação ao tempo inicial tanto para o teor de compostos fenólicos quanto para a atividade antioxidante durante o armazenamento. De modo geral, a diminuição da atividade antioxidante está correlacionada com a degradação dos compostos fenólicos, compostos em maior concentração no suco. Este comportamento também foi observado por Agcam et al. (2014) durante a avaliação do armazenamento, sob refrigeração, do suco de laranja pasteurizado, bem como por Guan et al. (2016) na avaliação do suco de manga pasteurizado e armazenado por 60 dias.

Segundo Laorko et al. (2013), a diminuição dos compostos fenólicos ao longo do armazenamento ocorre, provavelmente, pela oxidação seguida da reação de polimerização destes compostos. Estes autores, ao avaliarem o efeito da temperatura de armazenamento $\left(4^{\circ} \mathrm{C}, 27^{\circ} \mathrm{C} \mathrm{e}\right.$ $37^{\circ} \mathrm{C}$ ) nas características químicas do suco de abacaxi clarificado por microfiltração, observaram uma redução significativa desses compostos bioativos em todas as temperaturas e, como esperado, a menor redução (11,2\%) se deu também na temperatura mais baixa $\left(4^{\circ} \mathrm{C}\right)$, na qual a cinética de decomposição é mais lenta.

\section{Conclusão}

O estudo realizado mostrou que a combinação da pasteurização com o armazenamento sob refrigeração a $6{ }^{\circ} \mathrm{C}$ foi adequada para a estabilização física, química e microbiológica do suco de umbu por 90 dias de armazenamento. 
Avaliação do armazenamento a frio sobre os compostos bioativos e as características físico-químicas e microbiológicas do suco de umbu pasteurizado

Ribeiro, L. O. et al.

\section{Agradecimentos}

Os autores agradecem ao CNPq pelo apoio financeiro para a realização deste trabalho e também às instituições UFRJ e Embrapa Agroindústria de alimentos.

\section{Referências}

AGCAM, E.; AKYILDIZ, A.; EVRENDILEK, G. A. Comparison of phenolic compounds of orange juice processed by pulsed electric fields (PEF) and conventional thermal pasteurisation. Food Chemistry, London, v. 143, p. 354-361, 2014.

AMERICAN PUBLIC HEALTH ASSOCIATION - APHA. Compendium of methods for the microbiological examination of foods. 4. ed. Washington: APHA, 2001. 676 p.

BRASIL. Ministério da Agricultura, Pecuária e Abastecimento. Instrução Normativa n 01, de 07 de janeiro de 2000. Dispõe do Padrão de Identidade e Qualidade de polpas de frutas. Diário Oficial [da] República Federativa do Brasil, Brasília, DF, 10 jan. 2000.

BRASIL. Ministério da Saúde. Agência Nacional de Vigilância Sanitária. Resolução RDC n¹2, de 02 de janeiro de 2001. Dispõe sobre os princípios gerais para o estabelecimento de critérios e padrões microbiológicos para alimentos. Diário Oficial [da] República Federativa do Brasil, Brasília, DF, 10 jan. 2001.

FELLOWS, P. J. Tecnologia do processamento de alimentos: princípio e prática. 2. ed. Porto Alegre: Artmed, 2006. 602 p.

FERREIRA, J. E. M.; RODRIGUEZ-AMAYA, D. B. Degradation of lycopene and beta-carotene in model systems and in lyophilized guava during ambient storage: kinetics, structure, and matrix effects. Journal of Food Science, Chicago, v. 78, n. 8, p. 589-594, 2008. PMid:19019101. http://dx.doi.org/10.1111/j.17503841.2008.00919.x

FERREIRA, V. L. P. Princípios e aplicações da colorimetria em alimentos. Campinas: ITAL, 1981. 86p.

GAMA, J. J. T.; SYLOS, C. M. Effect of thermal pasteurization and concentration on carotenoid composition of Brazilian Valencia orange juice. Food Chemistry, London, v. 100, n. 4, p. 16861690, 2007.

GEORGÉ, S.; BRAT, P.; ALTER, P.; AMIOT, M. J. Rapid determination of polyphenols and vitamin $C$ in plant-derived products. Journal of Agricultural and Food Chemistry, Easton, v. 53, n. 5, p. 1370-1373, 2005. PMid:15740008. http://dx.doi.org/10.1021/ jf048396b.

GODOY, R. L. O.; NASCIMENTO, E. M.; RIBEIRO, L. O.; NASCIMENTO, L. S. M.; PACHECO, S.; BORGUINI, R. G.; MATTA, V. M. Metodologia para identificação e quantificação de flavonoides na polpa de umbu por cromatografia líquida de alta eficiência. Rio de Janeiro: Embrapa Agroindústria de Alimentos, 2013. p. 1-5. (Embrapa Agroindústria de Alimentos. Comunicado Técnico, 196).
GUAN, Y.; ZHOU, L.; BI, J.; YI, J.; LIU, X.; CHEN, Q.; WU, X.; $Z \mathrm{HOU}, \mathrm{M}$. Change of microbial and quality attributes of mango juice treated by high pressure homogenization combined with moderate inlet temperatures during storage. Innovative Food Science and Emerging Technologies, Berlin, v. 36, p. 320-329, 2016.

HORWITZ, W. (Ed.). Official methods of analysis of the Association of Official Analytical Chemists. Washington, D.C.: AOAC, 2000.

HORWITZ, W. (Ed.). Official methods of analysis of the Association of Official Analytical Chemists. Arlington: AOAC, 2005.

IGNAT, I.; VOLF, I.; POPA, V. I. A critical review of methods for characterisation of polyphenolic compounds in fruits and vegetables. Food Chemistry, London, v. 126, n. 4, p. 18211835, 2011.

INSTITUTO BRASILEIRO DE GEOGRAFIA E ESTATÍSTICA - IBGE. Produção extrativa vegetal. Rio de Janeiro: IBGE, 2014. Disponível em: <http://www.ibge.gov.br>. Acesso em: 01 nov. 2014.

KEENAN, D. F.; TIWARI, B. K.; PATRAS, A.; GORMLEY, R.; BUTLER, F.; BRUNTON, N. P. Effect of sonication on the bioactive, quality and rheological characteristics of fruit smoothies. International Journal of Food Science and Technology, London, v. 47, n. 4, p. 827-836, 2012. http://dx.doi.org/10.1111/j.1365-2621.2011.02915.x.

LAORKO, A.; TONGCHITPAKDEE, S.; YOURAVONG, W. Storage quality of pineapple juice non-thermally pasteurized and clarified by microfiltration. Journal of Food Engineering, Essex, v. 116, n. 2, p. 554-561, 2013. http://dx.doi.org/10.1016/j. jfoodeng.2012.12.033.

MATTIETTO, R. A.; LOPES, A. S.; MENEZES, H. C. Estabilidade do néctar misto de cajá e umbu. Ciência e Tecnologia de Alimentos, Campinas, v. 27 n. 3, p. 456-463, 2007

MENA, P.; VEGARA, S.; MARTÍ, N.; GARCÍA-VIGUERA, C.; SAURA, D.; VALERO, M. Changes on indigenous microbiota, colour, bioactive compounds and antioxidant activity of pasteurised pomegranate juice. Food Chemistry, London, v. 141, n. 3, p. 2122-2129, 2013. PMid:23870937. http://dx.doi.org/10.1016/j. foodchem.2013.04.118.

NACZK, M.; SHAHIDI, F. Extraction and analysis of phenolics in food. Journal of Chromatography A, Amsterdam, v. 1054, n. 1-2, p. 95-111, 2004.

NACZK, M.; SHAHIDI, F. Phenolics in cereals, fruits and vegetables: occurrence, extraction and analysis. Journal of Pharmaceutical and Biomedical Analysis, Amsterdam, v. 41, n. 5, p. 1523-1542, 2006. PMid:16753277. http://dx.doi. org/10.1016/j.jpba.2006.04.002.

OLIVEIRA, A.; PINTADO, M.; ALMEIDA, D. P. F. Phytochemical composition and antioxidant activity of peach as affected by pasteurization and storage duration. LWT - Food Science and Technology, Avignon, v. 49, n. 2, p. 202-207, 2012. 
Avaliação do armazenamento a frio sobre os compostos bioativos e as características físico-químicas e microbiológicas do suco de umbu pasteurizado

Ribeiro, L. O. et al.

OLIVEIRA, E. A.; BORGES, S. V.; FURTADO, A. A. L.; MODESTA, R. C. D.; GODOY, R. O. Heat processing (HTST) of umbu (Spondias tuberosa Arruda Câmara) pulp. Ciência e Tecnologia de Alimentos, Campinas, v. 31, n. 4, p. 923-928, 2011.

PACHECO, S.; PEIXOTO, F. M.; BORGUINI, R. G.; BOBEDA, C. R. R.; SANTIAGO, M. C. P. A.; GODOY, R. L. O. Microscale extraction method for HPLC carotenoid analysis in vegetable matrices. Scientia Agricola, Piracicaba, v. 71, n. 5, p. 345-355, 2014.

PAULA, B.; CARVALHO FILHO, C. D. C.; MATTA, V. M.; MENEZES, J. S.; LIMA, P. C.; PINTO, C. O.; CONCEIÇÃO, L. E. M. G. Produção e caracterização físico-química de fermentado de umbu. Ciência Rural, Santa Maria, v. 42, n. 9, p. 1688-1693, 2012. http://dx.doi.org/10.1590/S0103-84782012000900027.

RE, R.; PELlegrini, N.; PROTEGgente, A.; PANNALA, A.; YANG, M.; RICEEVANS, C. A. Antioxidant activity applying and improved ABTS radical cation decolorization assay. Free Radical Biological and Medicine, New York, v. 26, n. 9-10, p. 1231-1237, 1999.

REETZ, E. R.; KIST, B. B.; SANTOS, C. E.; CARVALHO, C.; DRUM, M. Brazilian fruit yearbook 2014. Santa Cruz do Sul: Gazeta Santa Cruz, 2015.

RIBEIRO, L. O. Avaliação de processos para a conservação de suco de umbu e de seus compostos bioativos. 2014. 113 f. Dissertação (Mestrado em Tecnologia de Processos Químicos e Bioquímicos)-Universidade Federal do Rio de Janeiro, Rio de Janeiro, 2014.

RODRIGUEZ-AMAYA, D. B. A guide to carotenoid analysis in foods. Washington: ILSI Press, 1999. 64 p.

SANTHIRASEGARAM, V.; RAZALI, Z.; GEORGE, D. S.; SOMASUNDRAM, C. Comparison of UV-C treatment and thermal pasteurization on quality of Chokanan mango (Mangifera indica L.) juice. Food and Bioproducts Processing, Rugby, v. 94, p. 313-321, 2015.

SINGLETON, V. L.; ROSSI, J. A. Colorimetry of total phenolics with phosphomolybdic-phosphotungstic acid reagents. American Journal of Enology and Viticulture, Davis, v. 16, n. 3, p. 144-168, 1965.

TSIMIDOU, M.; BILIADERIS, G. C. Kinect studies of saffron (Crocus sativus L.) quality deterioration. Journal Agricultural and Food Chemistry, Easton, v. 45, n. 8, p. 2890-2898, 1997.

XU, Z.; LIN, T.; WANG, Y.; LIAO, X. Quality assurance in pepper and orange juice blend treated by high pressure processing and high temperature short time. Innovative Food Science \& Emerging Technologies, Berlin, v. 31, p. 28-36, 2015. 